\title{
An application of the voter model-super-Brownian motion invariance principle
}

\author{
J. Theodore Cox ${ }^{a}$, Edwin A. Perkins ${ }^{b}$ \\ a Syracuse University, Department of Mathematics, New York, NY 13244-1150, USA \\ ${ }^{\mathrm{b}}$ The University of British Columbia, Canada \\ Received 25 July 2002; accepted 28 January 2003
}

\begin{abstract}
We show that the asymptotics for the hitting time of 0 of the voter model started from a single 1 can be obtained from the invariance principle for voter models and super-Brownian motion.

(c) 2003 Elsevier SAS. All rights reserved.
\end{abstract}

\section{Résumé}

Nous considérons le modèle du votant commençant avec un seul 1. Nous montrons qu'asymptotiquement le temps d'absorption en 0 peut être obtenu à partir du principe d'invariance liant le modèle du votant au super-mouvement brownien.

(c) 2003 Elsevier SAS. All rights reserved.

MSC: primary $60 \mathrm{~K} 35,60 \mathrm{G} 57$; secondary $60 \mathrm{~F} 05,60 \mathrm{~J} 80$

Keywords: Voter model; Super-Brownian motion

\section{Introduction and summary}

The voter model (see Chapter IV of [6]) is one of the simplest interacting particle systems. It has been studied extensively since the 1970's. An invariance principle has recently been established (see [2] and [4]) which shows that appropriately rescaled voter models converge weakly to super-Brownian motion. Our purpose here is to use this invariance principle to give a new proof of a fundamental result of Bramson and Griffeath (see [3]) on the asymptotic behavior of the voter model started from a single 1.

E-mail address: jtcox@syr.edu (J.T. Cox).

0246-0203/\$ - see front matter (c) 2003 Elsevier SAS. All rights reserved. doi:10.1016/j.anihpb.2003.01.001 
We begin by describing the voter model. Let $\xi_{t}$ denote the rate- 1 voter model on $\mathbf{Z}^{d}$ with voting $\operatorname{kernel} p(x, y)$ satisfying

$$
\begin{aligned}
& p(x, y)=p(0, y-x) \text { is irreducible and symmetric, with } p(0,0)=0, \\
& \text { and for some } 0<\sigma^{2}<\infty, \sum_{x \in \mathbf{Z}^{d}} p(0, x) x^{i} x^{j}=\delta(i, j) \sigma^{2}
\end{aligned}
$$

$\left(\delta(i, j)=1\right.$ for $i=j$, and $\delta(i, j)=0$ otherwise). We think of $\xi_{t}(x)$ as the opinion, either 0 or 1 , of a voter at site $x$ at time $t$, where the dynamics of $\xi_{t}$ are given by: independently, at each site $x$,

$$
\begin{aligned}
& 0 \rightarrow 1 \text { at rate } \sum_{y} p(x, y) 1_{\left\{\xi_{t}(y)=1\right\}}, \\
& 1 \rightarrow 0 \text { at rate } \sum_{y} p(x, y) 1_{\left\{\xi_{t}(y)=0\right\}} .
\end{aligned}
$$

We identify $\xi_{t}$ with the set $\left\{x: \xi_{t}(x)=1\right\}$, and let $\xi_{t}^{A}$ denote the voter model starting from 1 's exactly on $A$, $\xi_{0}^{A}=A$. We write $\xi_{t}^{x}$ for $\xi_{t}^{\{x\}}$, and make use of the usual additive construction of the voter model (see Section III.6 $[6])$,

$$
\xi_{t}^{A}=\bigcup_{x \in A} \xi_{t}^{x}
$$

It is easy to see that $\left|\xi_{t}^{0}\right|=\sum_{x} \xi_{t}^{0}(x)$ is a martingale, and that $\left|\xi_{t}^{0}\right|$ hits 0 eventually with probability one. Letting $p_{t}=P\left(\left|\xi_{t}^{0}\right|>0\right)$, it follows that $p_{t} \rightarrow 0$ as $t \rightarrow \infty$. Determination of the rate at which $p_{t} \rightarrow 0$ is not simple, since the rate at which $\left|\xi_{t}^{0}\right|$ changes depends on the spatial configuration of the set $\xi_{t}^{0}$. In the one-dimensional nearest neighbor case, $\xi_{t}^{0}$ is always an interval, and it is straightforward to determine the asymptotic behavior of $p_{t}$. In higher dimensions, even in the nearest neighbor case, the situation is far more complicated. Nevertheless, Bramson and Griffeath in [3] were able to obtain precise asymptotics.

To state their results, define (for $t>0$ )

$$
m_{t}= \begin{cases}t / \log t & \text { in } d=2, \\ t & \text { in } d \geqslant 3,\end{cases}
$$

let $\gamma_{2}=2 \pi \sigma^{2}$, and for $d \geqslant 3$, let $\gamma_{d}$ be the probability that a random walk with jump kernel $p(x, y)$ starting at the origin never returns to the origin. The notation $f(t) \sim g(t)$ as $t \rightarrow \infty$ means that $\lim _{t \rightarrow \infty} f(t) / g(t)=1$. Here is the Bramson and Griffeath result.

Theorem 1. Assume $d \geqslant 2$. As $t \rightarrow \infty$,

$$
p_{t} \sim 1 / \gamma_{d} m_{t}
$$

and

$$
P\left(p_{t}\left|\xi_{t}^{0}\right|>u|| \xi_{t}^{0} \mid>0\right) \rightarrow \mathrm{e}^{-u}, \quad u>0
$$

(Although the proof given in [3] was for the nearest-neighbor case $p(0, x)=(1 / 2 d)$ for $|x|=1$, as noted in Lemma 2 of [2], it is easily modified to cover kernels $p(x, y)$ satisfying (1.1).)

The asymptotics in Theorem 1 have proved to be important tools in the study of the voter model and its variants. There were two key ingredients in Bramson and Griffeath's proof. The first was their derivation of the upper bound

$$
p_{t}=\mathrm{O}\left(\frac{1}{m_{t}}\right) \quad \text { as } t \rightarrow \infty
$$


The second was Theorem 1.1 of [8], which gave asymptotics for the "patch of the origin" for a general stepping stone model. The proof of Sawyer's remarkable theorem proceded via the method of moments, using intricate calculations of transforms of coalescing random walk probabilites. It gave little insight into the theorem's conclusions. By combining the upper bound (1.5) and Sawyer's theorem, Bramson and Griffeath obtained (1.3) and (1.4).

Our purpose here is to give a new proof of these asymptotics which we feel is more probabilistic in nature and gives greater insight into why they hold. We make use of the upper bound (1.5), but avoid the use of Sawyer's result. Instead, we show that these asymptotics follow from an invariance principle showing that rescaled voter models converge to super-Brownian motion. (Unfortunately, it does not appear that the upper bound (1.5) can be obtained from this invariance principle.)

We begin by defining rescaled voter models $\xi_{t}^{N}$, which are rate- $N$ voter models on $\mathbf{S}_{\mathbf{N}}=\mathbf{Z}^{d} / \sqrt{N}$ with voting kernels $p_{N}(x, y)=p(x \sqrt{N}, y \sqrt{N})$ for $x, y \in \mathbf{S}_{\mathbf{N}}$. We assume throughout that $\left|\xi_{0}^{N}\right|<\infty$. Let $X_{t}^{N}$ denote the associated measure-valued processes

$$
X_{t}^{N}=\frac{1}{m_{N}} \sum_{x \in \xi_{t}^{N}} \delta_{x},
$$

where $\delta_{x}$ is the unit point mass as $x$.

Now let $X_{t}$ denote super-Brownian motion with branching rate $\gamma=2 \gamma_{d}$ and diffusion coefficient $\sigma^{2}$, taking values in $\mathcal{M}_{F}\left(\mathbf{R}^{d}\right)$, the space of finite measures on $\mathbf{R}^{d} . X_{t}$ is obtained as the limit of rescaled critical branching random walks or Brownian motions, and can be defined via the following martingale problem (see [7]): for all $\phi \in C_{0}^{\infty}\left(\mathbf{R}^{d}\right)$,

$$
M_{t}(\phi)=X_{t}(\phi)-X_{0}(\phi)-\int_{0}^{t} X_{s}\left(\frac{\sigma^{2} \Delta \phi}{2}\right) d s
$$

is a continuous $L^{2}$ martingale with $M_{0}(\phi)=0$ and square function

$$
\langle M(\phi)\rangle_{t}=\int_{0}^{t} X_{s}\left(\gamma \phi^{2}\right) d s
$$

(For a measure $\mu$ on $\mathbf{R}^{d}, \mu(\phi)=\int \phi(x) \mu(d x)$.)

We will make use of the explicit formulas

$$
P\left(X_{t}(\mathbf{1})>0\right)=1-\exp \left(-2 X_{0}(\mathbf{1}) / \gamma t\right)
$$

and

$$
E \exp \left(-\theta X_{t}(\mathbf{1})\right)=\exp \left(-\frac{2 \theta X_{0}(\mathbf{1})}{2+\theta \gamma t}\right),
$$

where $\mathbf{1}$ is the function identically 1 on $\mathbf{R}^{d}$. These formulas are not difficult to derive, since the total mass process, $X_{t}(\mathbf{1})$, is a Feller diffusion (see (II.5.11) and (II.5.12) of [7]).

Here is the invariance principle, Theorem 1.2 of [4]. The symbol $\Rightarrow$ denotes weak convergence, and $D\left(\mathbf{R}_{+}, \mathcal{M}_{F}\left(\mathbf{R}^{d}\right)\right)$ is the Skorohod space of cadlag $\mathcal{M}_{F}\left(\mathbf{R}^{d}\right)$-valued paths.

Theorem 2. Assume $d \geqslant 2$, and $X_{0}^{N} \rightarrow X_{0} \in \mathcal{M}_{F}\left(\mathbf{R}^{d}\right)$ as $N \rightarrow \infty$. Then $X_{\bullet}^{N} \Rightarrow X_{\bullet}$ in $D\left(\mathbf{R}_{+}, \mathcal{M}_{F}\left(\mathbf{R}^{d}\right)\right)$.

Let us consider the case $d \geqslant 3$ and see why Theorem 2 and the formulas (1.6) and (1.7) suggest that (1.3) and (1.4) should hold. Let $\mathcal{L}$ denote law, and let $\approx$ denote "approximate equality". Let $\xi_{0}^{N}=\{0\}$. Then 
$\mathcal{L}\left(\left|\xi_{t}^{0}\right|\right)=\mathcal{L}\left(\left|\xi_{t / N}^{N}\right|\right)$, and in view of Theorem 2, we expect that $\mathcal{L}\left(\left|\xi_{t}^{N}\right|\right) \approx \mathcal{L}\left(N X_{t}(\mathbf{1})\right)$ for large $N$, where $X_{0}(\mathbf{1})=\left|\xi_{0}^{N}\right| / N=1 / N$. Setting $t=1$ and using (1.6), it follows that

$$
p_{N}=P\left(\left|\xi_{N}^{0}\right|>0\right) \approx P\left(N X_{1}(\mathbf{1})>0\right)=1-\exp \left(-1 / N \gamma_{d}\right) \sim 1 / N \gamma_{d}
$$

as $N \rightarrow \infty$. This is (1.3). Similarly, for $\theta>0$, we have

$$
\begin{aligned}
E\left(1-\mathrm{e}^{-\theta p_{N}\left|\xi_{N}^{0}\right|}|| \xi_{N}^{0} \mid>0\right) & =p_{N}^{-1} E\left(1-\mathrm{e}^{-\theta p_{N}\left|\xi_{N}^{0}\right|}\right) \\
& \approx p_{N}^{-1} E\left(1-\mathrm{e}^{-\theta p_{N} N X_{1}(\mathbf{1})}\right) \\
& =p_{N}^{-1}\left(1-\exp \left(-\frac{\theta p_{N}}{1+\theta N p_{N} \gamma_{d}}\right)\right),
\end{aligned}
$$

where we have used (1.7) and the fact that $X_{0}(\mathbf{1})=1 / N$. It is easy to see, since $p_{N} \sim 1 / N \gamma_{d}$ as $N \rightarrow \infty$, that the last expression converges to $\theta /(1+\theta)$, which implies (1.4).

In order to make these arguments rigorous, we make use of the upper bound (1.5) and ideas from [2]. We also require a corollary to Theorem 2, which says that the hitting times of 0 for $X_{t}^{N}$ converge weakly to the hitting time of 0 for $X_{t}$. With these ingredients, we give a proof of Theorem 1 which avoids the use of Sawyer's theorem.

We close the introduction by stating our hitting time result. For $a \geqslant 0$ let $\tau_{a}^{N}$ and $\tau_{a}$ be the hitting times

$$
\tau_{a}^{N}=\inf \left\{t>0: X_{t}^{N}(\mathbf{1}) \leqslant a\right\} \quad \text { and } \tau_{a}=\inf \left\{t>0: X_{t}(\mathbf{1}) \leqslant a\right\} .
$$

Corollary 3. Assume that $d \geqslant 2$, and $X_{0}^{N} \rightarrow X_{0} \in \mathcal{M}_{F}\left(\mathbf{R}^{d}\right)$. Then

$$
\lim _{N \rightarrow \infty} P\left(\tau_{0}^{N}>t\right)=P\left(\tau_{0}>t\right)=1-\exp \left(-\frac{X_{0}(\mathbf{1})}{t \gamma_{d}}\right), \quad t>0 .
$$

The reason that Corollary 3 does not follow immediately from Theorem 2 is that there is no "soft" way to ensure that once $X_{t}^{N}(\mathbf{1})$ reaches a level $a>0$ very close to 0 , it doesn't linger there rather than reaching 0 fairly quickly. We use (1.5) to take care of this problem.

\section{Proofs}

We first prove Corollary 3, then Theorem 1.

Proof of Corollary 3. The second equality is immediate from (1.6), so we only need prove the first equality. For $t>0$ define

$$
I_{t}^{N}=\inf \left\{X_{s}^{N}(\mathbf{1}): 0 \leqslant s \leqslant t\right\}, \quad I_{t}=\inf \left\{X_{s}(\mathbf{1}): 0 \leqslant s \leqslant t\right\} .
$$

It follows from Theorem 2 that $X_{\bullet}^{N}(\mathbf{1}) \Rightarrow X_{\bullet}(\mathbf{1})$ as $N \rightarrow \infty$, and since the infimum over a path is a continuous function on the space of continuous paths, we also have, for fixed $t>0, I_{t}^{N} \Rightarrow I_{t}$. More specifically, as $X_{\bullet}(\mathbf{1})$ is continuous, this follows from Theorem 3.10.2 of [5]. For any $a \geqslant 0,\left\{I_{t}^{N}>a\right\}=\left\{\tau_{a}^{N}>t\right\}$ and $\left\{I_{t}>a\right\}=\left\{\tau_{a}>t\right\}$. Consequently,

$$
\liminf _{N \rightarrow \infty} P\left(\tau_{0}^{N}>t\right) \geqslant P\left(\tau_{0}>t\right) .
$$

By (1.5) there is a constant $C$ such that $p_{t} \leqslant C / m_{t}$. Consequently, for any initial state $\xi_{0}^{N}$,

$$
P\left(\xi_{t}^{N}>0\right) \leqslant C\left|\xi_{0}^{N}\right| / m_{N t} .
$$


This follows from the additive construction of the voter model, since

$$
P\left(\xi_{t}^{N}>0\right)=P\left(\left|\bigcup_{x \in \xi_{0}^{N}} \xi_{t}^{N, x}\right|>0\right) \leqslant \sum_{x \in \xi_{0}^{N}} P\left(\left|\xi_{t}^{N, x}\right|>0\right) \leqslant C\left|\xi_{0}^{N}\right| / m_{N t} .
$$

Now choose $s, a$ such that $s<t$ and $0<a<X_{0}$ (1). Then, making use of (2.2) and the strong Markov property at time $\tau_{a}^{N}$, for $N$ large enough so that $X_{0}^{N}(\mathbf{1})>a$, we have

$$
\begin{aligned}
P\left(\tau_{0}^{N}>t\right) & \leqslant P\left(\tau_{a}^{N}>s\right)+P\left(\tau_{a}^{N} \leqslant s, \tau_{0}^{N}>t\right) \\
& \leqslant P\left(\tau_{a}^{N}>s\right)+\sup \left\{P\left(\left|\xi_{t-s}^{N}\right|>0\right):\left|\xi_{0}^{N}\right| \leqslant a m_{N}\right\} \\
& \leqslant P\left(\tau_{a}^{N}>s\right)+\operatorname{Cam}_{N} / m_{N(t-s)} \\
& =P\left(I_{s}^{N}>a\right)+\operatorname{Cam}_{N} / m_{N(t-s)} .
\end{aligned}
$$

We now take $a$ to be a continuity point for the distribution function of $I_{s}$, so that $P\left(I_{s}^{N}>a\right) \rightarrow P\left(I_{s}>a\right)$ as $N \rightarrow \infty$. Since $P\left(I_{s}>a\right)=P\left(\tau_{a}>s\right) \leqslant P\left(\tau_{0}>s\right)$, using the definition of $m_{t}$ we therefore have

$$
\limsup _{N \rightarrow \infty} P\left(\tau_{0}^{N}>t\right) \leqslant P\left(\tau_{0}>s\right)+C a /(t-s) .
$$

We may now let $s \uparrow t$ and $a \downarrow 0$ such that $a /(t-s) \rightarrow 0$, to obtain (recall from (1.6) that $\tau_{0}$ has a continuous distribution function)

$$
\limsup _{N \rightarrow \infty} P\left(\tau_{0}^{N}>t\right) \leqslant P\left(\tau_{0}>t\right) .
$$

Together, (2.1) and (2.3) imply (1.8).

Proof of Theorem 1. For $\varepsilon>0$, let $B_{N, \varepsilon}$ be the box in $\mathbf{S}_{\mathbf{N}}$ centered at the origin of side length $\left(\varepsilon m_{N}\right)^{1 / d} / N^{1 / 2}$, so that $\left|B_{N, \varepsilon}\right| \sim \varepsilon m_{N}$ as $N \rightarrow \infty$. Let $\xi_{t}^{N, B_{N, \varepsilon}}$ denote the rate- $N$ voter model with $\xi_{0}^{N, B_{N, \varepsilon}}=B_{N, \varepsilon}$, with corresponding measure-valued process $X_{t}^{N, \varepsilon}$, and let $\xi_{t}^{N, x}$ denote the process with initial state $\xi_{0}^{N, x}=\{x\}$. Let $X_{t}^{\varepsilon}$ denote super-Brownian motion with $X_{0}=\varepsilon \delta_{0}$, branching rate $\gamma=2 \gamma_{d}$, and diffusion coefficient $\sigma^{2}$. Since $X_{0}^{N, \varepsilon} \rightarrow X_{0}^{\varepsilon}$, by Corollary 3 it follows that

$$
\lim _{N \rightarrow \infty} P\left(\left|\xi_{t}^{N, B_{N, \varepsilon}}\right|>0\right)=P\left(X_{t}^{\varepsilon}>0\right)=1-\mathrm{e}^{-\varepsilon / \gamma_{d} t} .
$$

Since

$$
P\left(\left|\xi_{t}^{N, B_{N, \varepsilon}}\right|>0\right) \leqslant \sum_{x \in B_{N, \varepsilon}} P\left(\left|\xi_{t}^{N, x}\right|>0\right)=\left|B_{N, \varepsilon}\right| P\left(\left|\xi_{t}^{N, 0}\right|>0\right)
$$

it follows that

$$
\liminf _{N \rightarrow \infty} m_{N} P\left(\left|\xi_{t}^{N, 0}\right|>0\right) \geqslant \frac{1-\mathrm{e}^{-\varepsilon / \gamma_{d} t}}{\varepsilon} .
$$

Letting $\varepsilon \rightarrow 0$, we obtain

$$
\liminf _{N \rightarrow \infty} m_{N} P\left(\left|\xi_{t}^{N, 0}\right|>0\right) \geqslant 1 / \gamma_{d} t .
$$

For a bound in the other direction, we appeal to additivity and inclusion-exclusion,

$$
P\left(\left|\xi_{t}^{N, B_{N, \varepsilon}}\right|>0\right) \geqslant \sum_{x \in B_{N, \varepsilon}} P\left(\left|\xi_{t}^{N, x}\right|>0\right)-\sum_{\substack{x \neq y \\ x, y \in B_{N, \varepsilon}}} P\left(\left|\xi_{t}^{N, x}\right|>0,\left|\xi_{t}^{N, y}\right|>0\right) .
$$


By a correlation inequality, Lemma 1 of [1], for $x \neq y$,

$$
P\left(\left|\xi_{t}^{N, x}\right|>0,\left|\xi_{t}^{N, y}\right|>0\right) \leqslant P\left(\left|\xi_{t}^{N, x}\right|>0\right) P\left(\left|\xi_{t}^{N, y}\right|>0\right)=P\left(\left|\xi_{t}^{N, 0}\right|>0\right)^{2} .
$$

It therefore follows that

$$
P\left(\left|\xi_{t}^{N, B_{N, \varepsilon}}\right|>0\right) \geqslant\left|B_{N, \varepsilon}\right| P\left(\left|\xi_{t}^{N, 0}\right|>0\right)-\left|B_{N, \varepsilon}\right|^{2} P\left(\left|\xi_{t}^{N, 0}\right|>0\right)^{2} .
$$

Rearranging this inequality and using the bound $p_{t} \leqslant C / m_{t}$, we obtain

$$
\left|B_{N, \varepsilon}\right| P\left(\left|\xi_{t}^{N, 0}\right|>0\right) \leqslant P\left(\left|\xi_{t}^{N, B_{N, \varepsilon}}\right|>0\right)+\left|B_{N, \varepsilon}\right|^{2} \frac{C^{2}}{m_{t N}^{2}} .
$$

Since $\left|B_{N, \varepsilon}\right| \sim \varepsilon m_{N}$ and $m_{N} / m_{N t} \rightarrow 1 / t$ as $N \rightarrow \infty$, (2.4) implies that

$$
\limsup _{N \rightarrow \infty} m_{N} P\left(\left|\xi_{t}^{N, 0}\right|>0\right) \leqslant \frac{1-\mathrm{e}^{-\varepsilon / \gamma_{d} t}}{\varepsilon}+C^{2} \varepsilon / t^{2} .
$$

Letting $\varepsilon \rightarrow 0$ now gives

$$
\limsup _{N \rightarrow \infty} m_{N} P\left(\left|\xi_{t}^{N, 0}\right|>0\right) \leqslant 1 / \gamma_{d} t .
$$

Together, (2.5) and (2.7) imply $m_{N} P\left(\left|\xi_{t}^{N, 0}\right|>0\right) \rightarrow 1 / \gamma_{d} t$ as $N \rightarrow \infty$. Setting $t=1$ we obtain (1.3).

To prove (1.4), we fix $\theta>0$ and set $\psi(u)=1-\mathrm{e}^{-\theta u}, u \geqslant 0$. We will use several times without comment the simple fact that $\psi(0)=0$. By Theorem 2,

$$
\lim _{N \rightarrow \infty} E \psi\left(X_{t}^{N, \varepsilon}(\mathbf{1})\right)=E \psi\left(X_{t}^{\varepsilon}(\mathbf{1})\right) .
$$

In view of (1.7), this shows that

$$
\lim _{N \rightarrow \infty} E \psi\left(\frac{\left|\xi_{t}^{N, B_{N, \varepsilon}}\right|}{m_{N}}\right)=1-\exp \left(-\frac{\theta \varepsilon}{1+\theta \gamma_{d} t}\right) .
$$

We will show that

$$
\left|B_{N, \varepsilon}\right| E \psi\left(\frac{\left|\xi_{t}^{N, 0}\right|}{m_{N}}\right)=E \psi\left(\frac{\left|\xi_{t}^{N, B_{N, \varepsilon}}\right|}{m_{N}}\right)+\mathrm{O}\left(\frac{\varepsilon^{2}}{t^{2}}\right) \quad \text { as } N \rightarrow \infty .
$$

By (2.8) and the fact that $p_{t N}\left|B_{N, \varepsilon}\right| \rightarrow \varepsilon / \gamma_{d} t$ as $N \rightarrow \infty$, (2.9) implies

$$
\limsup _{N \rightarrow \infty}\left|E\left(\psi\left(\frac{\left|\xi_{t}^{N, 0}\right|}{m_{N}}\right)|| \xi_{t}^{N, 0} \mid>0\right)-\gamma_{d} t \frac{1-\exp \left(-\theta \varepsilon /\left(1+\theta \gamma_{d} t\right)\right)}{\varepsilon}\right|=\mathrm{O}\left(\frac{\varepsilon}{t}\right) .
$$

Letting $\varepsilon \rightarrow 0$ gives

$$
\lim _{N \rightarrow \infty} E\left(\psi\left(\frac{\left|\xi_{t}^{N, 0}\right|}{m_{N}}\right)|| \xi_{t}^{N, 0} \mid>0\right)=\frac{\theta \gamma_{d} t}{1+\theta \gamma_{d} t}
$$

That is, conditional on $\left|\xi_{t}^{N, 0}\right|>0,\left|\xi_{t}^{N, 0}\right| / m_{N} \Rightarrow \gamma_{d} t \mathcal{E}(1)$, where $\mathcal{E}(1)$ denotes an exponential random variable with mean 1. Since $p_{t N} m_{N} \rightarrow 1 / \gamma_{d} t$, this implies that, conditional on $\left|\xi_{t}^{N, 0}\right|>0, p_{t N}\left|\xi_{t}^{N, 0}\right| \Rightarrow \mathcal{E}(1)$ as $N \rightarrow \infty$. This proves (1.4).

To prove (2.9), we introduce the set $S_{t}^{N}=\left\{x \in B_{N, \varepsilon}:\left|\xi_{t}^{N, x}\right|>0\right\}$ of surviving families in $\xi_{t}^{N, B_{N, \varepsilon}}$. Clearly,

$$
E \psi\left(\frac{\left|\xi_{t}^{N, \varepsilon}\right|}{m_{N}}\right)=E\left(\psi\left(\frac{\left|\xi_{t}^{N, \varepsilon}\right|}{m_{N}}\right) ;\left|S_{t}^{N}\right|=1\right)+E\left(\psi\left(\frac{\left|\xi_{t}^{N, \varepsilon}\right|}{m_{N}}\right) ;\left|S_{t}^{N}\right| \geqslant 2\right) .
$$


We use the correlation inequality (2.6) to handle the second term on the right side of (2.10). By additivity and the fact that $\psi(0)=0$,

$$
\begin{aligned}
E\left(\psi\left(\frac{\left|\xi_{t}^{N, \varepsilon}\right|}{m_{N}}\right) ;\left|S_{t}^{N}\right| \geqslant 2\right) & \leqslant P\left(\bigcup_{\substack{x \neq y \\
x, y \in B_{N, \varepsilon}}}\left\{\left|\xi_{t}^{N, x}\right|>0,\left|\xi_{t}^{N, y}\right|>0\right\}\right) \\
& \leqslant \sum_{\substack{x \neq y \\
x, y \in B_{N, \varepsilon}}} P\left(\left|\xi_{t}^{N, x}\right|>0,\left|\xi_{t}^{N, y}\right|>0\right) \\
& \leqslant\left|B_{N, \varepsilon}\right|^{2} P\left(\left|\xi_{t}^{N, 0}\right|>0\right)^{2} .
\end{aligned}
$$

Since the definition of $B_{N, \varepsilon}$ and (1.5) imply that

$$
\left|B_{N, \varepsilon}\right| p_{t N}=\mathrm{O}\left(\frac{\varepsilon}{t}\right) \quad \text { as } N \rightarrow \infty
$$

we have shown that

$$
E\left(\psi\left(\frac{\left|\xi_{t}^{N, \varepsilon}\right|}{m_{N}}\right) ;\left|S_{t}^{N}\right| \geqslant 2\right)=\mathrm{O}\left(\frac{\varepsilon^{2}}{t^{2}}\right) \quad \text { as } N \rightarrow \infty .
$$

Consider now the first term on the right side of (2.10). The event $\left\{\left|\xi_{t}^{N, x}\right|>0\right\}$ is the disjoint union $\left\{S_{t}^{N}=\right.$ $\{x\}\} \cup\left\{\left|\xi_{t}^{N, x}\right|>0,\left|S_{t}^{N}\right|>1\right\}$, and

$$
P\left(\left|\xi_{t}^{N, x}\right|>0,\left|S_{t}^{N}\right|>1\right) \leqslant \sum_{\substack{y \neq x \\ y \in B_{N, \varepsilon}}} P\left(\left|\xi_{t}^{N, x}\right|>0,\left|\xi_{t}^{N, y}\right|>0\right) \leqslant\left|B_{N, \varepsilon}\right| P\left(\left|\xi_{t}^{N, 0}\right|>0\right)^{2},
$$

where we have again used the inequality (2.6). Consequently,

$$
\begin{aligned}
E\left(\psi\left(\frac{\left|\xi_{t}^{N, B_{N, \varepsilon}}\right|}{m_{N}}\right) ;\left|S_{t}^{N}\right|=1\right)= & \sum_{x \in B_{N, \varepsilon}} E\left(\psi\left(\frac{\left|\xi_{t}^{N, x}\right|}{m_{N}}\right) ; S_{t}^{N}=\{x\}\right) \\
= & \sum_{x \in B_{N, \varepsilon}}\left[E\left(\psi\left(\frac{\left|\xi_{t}^{N, x}\right|}{m_{N}}\right) ;\left|\xi_{t}^{N, x}\right|>0\right)\right. \\
& \left.-E\left(\psi\left(\frac{\left|\xi_{t}^{N, x}\right|}{m_{N}}\right) ;\left|\xi_{t}^{N, x}\right|>0,\left|S_{t}^{N}\right|>1\right)\right] \\
= & \left|B_{N, \varepsilon}\right| E\left(\psi\left(\frac{\left|\xi_{t}^{N, 0}\right|}{m_{N}}\right)\right)+\mathrm{O}\left(\left(\left|B_{N, \varepsilon}\right| p_{N t}\right)^{2}\right) \\
= & \left|B_{N, \varepsilon}\right| E\left(\psi\left(\frac{\left|\xi_{t}^{N, 0}\right|}{m_{N}}\right)\right)+\mathrm{O}\left(\frac{\varepsilon^{2}}{t^{2}}\right)
\end{aligned}
$$

as $N \rightarrow \infty$, where (2.13) is used in the next to last equality and (2.11) is used in the last equality. Plugging (2.12) and (2.14) into (2.10) yields (2.9), and we are done.

\section{References}

[1] R. Arratia, Limiting point processes for rescaling of coalescing and annihilating random walks on $\mathbf{Z}^{d}$, Ann. Probab. 9 (1981) 909-936.

[2] M. Bramson, J.T. Cox, J.-F. Le Gall, Super-Brownian limits of voter model clusters, Ann. Probab. 29 (2001) 1001-1032. 
[3] M. Bramson, D. Griffeath, Asymptotics for interacting particle systems on $\mathbf{Z}^{d}$, Z. Wahrsch. Verw. Geb. 53 (1980) 183-196.

[4] J.T. Cox, R. Durrett, E.A. Perkins, Rescaled voter models converge to super-Brownian motion, Ann. Probab. 28 (2000) 185-234.

[5] S.N. Ethier, T.G. Kurtz, Markov Process: Characterization and Convergence, Wiley, New York, 1986.

[6] T.M. Liggett, Interacting Particle Systems, Springer, New York, 1985.

[7] E.A. Perkins, Dawson-Watanabe superprocesses and measure-valued diffusions, in: Lectures on Probability Theory and Statistics; École d'Eté des Probabilités de St. Flour XXIX, 1999, in: Lecture Notes in Math., vol. 1781, Springer, 2002.

[8] S. Sawyer, A limit theorem for patch sizes in a selectively-neutral migration model, J. Appl. Probab. 16 (1979) $482-495$. 